\title{
On the Preservation of Some Additional Properties via the Quadratic Polynomial Construction Method of Fuzzy Implication Functions
}

\author{
Isabel Aguiló ${ }^{a, b}$ and Sebastia Massanet ${ }^{a, b}$ and J. Vicente Riera ${ }^{a, b}$ \\ ${ }^{a}$ Department of Mathematics and Computer Science, University of the Balearic Islands, \\ Ctra. de Valldemossa, Km.7.5, E-07122 Palma de Mallorca, Spain, \\ \{isabel.aguilo, s.massanet, jvicente.riera\}@uib.es \\ ${ }^{b}$ Health Research Institute of the Balearic Islands (IdISBa), 07010 Palma, Spain
}

\begin{abstract}
Following the extensively studied line of research of proposing new construction methods of fuzzy implication functions, recently a construction method based on a quadratic polynomial function and a given fuzzy implication function was proposed. The importance of this method relies on the fact that some additional properties are preserved from the original fuzzy implication function to the generated one for some quadratic polynomial functions. In this paper, the preservation of two pairs of additional properties under this method is deeply studied providing some quadratic construction methods with this behaviour.
\end{abstract}

Keywords: Fuzzy implication function, quadratic polynomial construction, ordering property, left neutrality principle, contrapositive symmetry.

\section{Introduction}

Within the theoretical study of fuzzy implication functions, the search of new construction methods has been always a hot topic. The disposal of a great number of different models with different additional properties is necessary in order to choose the model which fits best the meaning and desired properties in each application (see [13]).

There are many construction methods. One of the strategies relies on the transformation of one or two given fuzzy implication functions to generate a new one. Among these methods, we can highlight the following ones: the upper, lower and medium contrapositivisations, the conjugation, the reciprocation and the maximum, minimum or convex combinations of fuzzy implication functions (see [4]), some methods based on new types of contrapositivisations [1], horizontal and vertical threshold generation methods $[8,9,10]$, the $F N I$-method [2, 12], the $\circledast$-composition $[14,15]$, among others. For an extensive compilation, we refer the reader to [3] and specially the chapter [11] for more details.

Although many construction methods can be proposed, researchers are always interested in methods which (i) provide operators with a simple expression and (ii) preserve the additional properties of the initial fuzzy implication function to the generated implication. These assets ensure that researchers can use the method to generate fuzzy implication functions which are not prone to numerical errors and which satisfy the desired additional properties.

Recently, a construction method based on quadratic polynomial functions was proposed in [7]. Starting from a polynomial $F:[0,1]^{3} \rightarrow \mathbb{R}$ and a fuzzy implication function $I$, a new fuzzy implication function is obtained by means of $I_{F}(x, y)=F(x, y, I(x, y))$. This method generates fuzzy implication functions with a simple expression, easy to implement in any application. In [7], a full characterization of those functions $F:[0,1]^{3} \rightarrow \mathbb{R}$ providing always a fuzzy implication function through the method was proved. Additionally, characterizations of quadratic functions preserving some specific additional properties were also presented. Indeed, results were presented for the preservation of the pairs (NP) and $\left(\mathbf{C P}\left(N_{c}\right)\right),(\mathbf{N P})$ and (IP), and (IP) and $\left(\mathbf{C P}\left(N_{c}\right)\right)$, see Section 2 for the details. However, many other important additional properties were not studied in [7]. In this paper, we will deal with the ordering property (OP) in conjunction with either (NP) or $\left(\mathbf{C P}\left(N_{c}\right)\right)$.

The structure of the paper is as follows. Section 2 includes some preliminaries about fuzzy implication functions and the additional properties which will be studied in the paper. Section 3 recalls the basic facts on the construction method based on quadratic poly- 
nomial functions. Then, Section 4 is devoted to finding quadratic constructions preserving the pairs (i) $\left(\mathbf{C P}\left(N_{c}\right)\right)$ and (OP), (ii) (NP) and (OP). Finally, the paper ends with Section 5 devoted to some conclusions and future work.

\section{Preliminaries}

Let us provide some definitions and results that will be used throughout this paper. First, we present the definition of fuzzy negation.

Definition 2.1 ([6, Definition 1.1]). A decreasing function $N:[0,1] \rightarrow[0,1]$ is a fuzzy negation, if $N(0)=$ 1 and $N(1)=0$. A fuzzy negation $N$ is

(i) strict, if it is continuous and strictly decreasing,

(ii) strong, if it is an involution, i.e., $N(N(x))=x$ for all $x \in[0,1]$

There are many different fuzzy negations. Maybe the most well-known is the classical negation given by $N_{c}(x)=1-x$ for all $x \in[0,1]$.

Next, we recall the definition of a fuzzy implication function.

Definition 2.2 ([6, Definition 1.15]). A binary function $I:[0,1]^{2} \rightarrow[0,1]$ is called a fuzzy implication function if it satisfies:

(I1) $I(x, z) \geq I(y, z)$ when $x \leq y$, for all $z \in[0,1]$.

(I2) $I(x, y) \leq I(x, z)$ when $y \leq z$, for all $x \in[0,1]$.

(I3) $I(0,0)=I(1,1)=1$ and $I(1,0)=0$.

Let us denote by $\mathscr{I}$ the class of all fuzzy implication functions. Note that from the definition, we can deduce that for all $I \in \mathscr{I}, I(0, x)=1$ and $I(x, 1)=1$ for all $x \in[0,1]$, while the symmetric values $I(x, 0)$ and $I(1, x)$ are not determined from the definition. We recall some additional properties of fuzzy implication functions which will be used in this work:

- The left neutrality principle,

$$
I(1, y)=y, \quad y \in[0,1] .
$$

- The identity principle,

$$
I(x, x)=1, \quad x \in[0,1] .
$$

- The ordering property,

$$
x \leq y \Longleftrightarrow I(x, y)=1, \quad x, y \in[0,1] .
$$

- The law of contraposition with respect to a fuzzy negation $N$,

$$
I(N(y), N(x))=I(x, y), \quad x, y \in[0,1], \quad(\mathbf{C P}(\mathbf{N}))
$$

and in particular with respect to the classical negation $N_{c}$,

$$
I(1-y, 1-x)=I(x, y), \quad x, y \in[0,1] . \quad\left(\mathbf{C P}\left(\mathbf{N}_{\mathbf{c}}\right)\right)
$$

Moreover, we can define the concept of the natural negation of a fuzzy implication function, which is always a fuzzy negation in the sense of Definition 2.1.

Definition 2.3. Let I be a fuzzy implication function. The function $N_{I}$ defined by $N_{I}(x)=I(x, 0)$ for all $x \in$ $[0,1]$ is called the natural negation of $I$.

As we have already mentioned in the introduction, there exist different construction methods of new fuzzy implication functions from given ones. One of them that will be important in the proofs of some of the results is recalled in the following proposition.

Proposition 2.1 ([4]). Let $I_{1}, I_{2}$ be two fuzzy implication functions and $\lambda \in[0,1]$. The binary function $I:[0,1]^{2} \rightarrow[0,1]$ given by

$I(x, y)=(1-\lambda) I_{1}(x, y)+\lambda I_{2}(x, y) \quad$ for all $x, y \in[0,1]$

is always a fuzzy implication function.

Fuzzy implication functions constructed as in Eq. (1) are called convex linear combinations of $I_{1}$ and $I_{2}$. Moreover, it is well known that this construction method preserves (NP), (IP), (OP) and $\left(\mathbf{C P}\left(N_{c}\right)\right)$, but in general, it does not preserve (EP) (see [4] for more details).

\section{Construction of Fuzzy Implication Functions from Polynomial Ternary Functions}

As it is aforementioned in the introduction, in [7] a method that allows to construct fuzzy implication functions from polynomial ternary functions is presented. The idea of this method is as follows. Let $F:[0,1]^{3} \rightarrow$ $\mathbb{R}$ be a ternary function whose expression is given by

$$
\begin{aligned}
F(x, y, z)= & a x^{2}+b y^{2}+c z^{2}+d x y+ \\
& e x z+f y z+g x+h y+i z+j,
\end{aligned}
$$

where coefficients $a, b, c, d, e, f, g, h, i, j$ are in $\mathbb{R}$ and let us consider a fuzzy implication function $I \in \mathscr{I}$. For all $x, y \in[0,1]$ let us take $z=I(x, y)$ and define the function $I_{F}:[0,1]^{2} \rightarrow \mathbb{R}$ by

$$
I_{F}(x, y)=F(x, y, I(x, y)) \quad \text { for all } x, y \in[0,1] .
$$


In this way, in [7] it is investigated when Expression (3) satisfies the conditions to be a fuzzy implication. An immediate example of this construction method is when we consider the projection on the third variable $F(x, y, z)=z$, which yields the original fuzzy implication function $I$. However, we want to emphasize that the resulting value $F(x, y, I(x, y))$ could be out of $[0,1]$. For instance, let us consider the Rescher implication

$$
I_{\mathbf{R S}}(x, y)= \begin{cases}1 & \text { if } x \leq y \\ 0 & \text { if } x>y\end{cases}
$$

and the ternary function $F(x, y, z)=x+y+z$. Thus, if we take $x=y=1$ it follows $F\left(1,1, I_{\mathbf{R S}}(1,1)\right)=3 \notin$ $[0,1]$.

In [7] a full characterization of those functions $F$ satisfying that $I_{F}$ is a fuzzy implication function for any given fuzzy implication $I$ is proved (see Theorem 3.1.). However, this is a very tough demand and many times we are interested only in obtaining fuzzy implication functions $I_{F}$ satisfying a concrete additional property for any fuzzy implication function $I$ satisfying that property. This is a key point because there is no relationship between the two results. Indeed, the set of functions $F$ which provide always a fuzzy implication function is not contained in the set of functions $F$ providing always a fuzzy implication function satisfying one concrete property (given that the initial implication also satisfies it), not vice versa. For further details, see Example 4.1 in [7].

Let us recall for the sake of completeness, two results of [7] which will be used in this paper. First, the function $I_{F}$ must satisfy the boundary conditions inherent to fuzzy implication functions for any $I \in \mathscr{I}$.

Proposition 3.1 ([7, Proposion 3.2]). Let $F: \mathbb{R}^{3} \rightarrow \mathbb{R}$ be a quadratic function of the form (2) and $I:[0,1]^{2} \rightarrow$ $[0,1]$ be a fuzzy implication function. Then the following statements are equivalent:

(i) $I_{F}$ fulfils the boundary conditions (I3) and $I_{F}(0, x)=I_{F}(x, 1)=1$ for all $x \in[0,1]$

(ii) $a=b=0, e=-(d+g), h=-f, j=-g$, and $i=1-c+g$. Moreover, in this case, the expression of $F$ is given by

$$
\begin{aligned}
& F(x, y, z)=c z^{2}+d x y-(d+g) x z+ \\
& f y z+g x-f y+(1-c+g) z-g
\end{aligned}
$$

with $c, d, g, f \in \mathbb{R}$.

As pointed out in the introduction, in [7] some characterizations of those quadratic constructions that preserve some desired additional properties such as (NP), (IP) or $\left(\mathbf{C P}\left(N_{c}\right)\right)$ are presented.
Proposition 3.2 ([7, Proposion 4.1]). Let $F:[0,1]^{3} \rightarrow$ $\mathbb{R}$ be a quadratic function of the form (4) and $I$ : $[0,1]^{2} \rightarrow[0,1]$ be a fuzzy implication function. Then the next properties hold:

(i) If I satisfies (NP), then $I_{F}$ fulfils (NP) if and only if $f+c=0$.

(ii) If I satisfies (IP), then $I_{F}$ fulfils (IP) if and only if $d=0$.

(iii) If I satisfies $\left(\mathbf{C P}\left(N_{c}\right)\right)$, then $I_{F}$ fulfils $\left(\mathbf{C P}\left(N_{c}\right)\right)$ if and only if $d+g-f=0$.

\section{On the Preservation of some Additional Properties}

In this section we will investigate those quadratic constructions that preserve at the same time two interesting additional properties from the initial given fuzzy implication function $I$ to the generated one $I_{F}$. First, we will analyze the pair formed by the left neutrality principle (NP) and the ordering property. Then, we will study the ordering property $(\mathbf{O P})$ and the contrapositive symmetry with respect to $N_{c}\left(\mathbf{C P}\left(\mathbf{N}_{\mathbf{c}}\right)\right.$.

\subsection{Quadratic constructions preserving (NP) and (OP)}

Let us study the quadratic constructions preserving (NP) and (OP).

Proposition 4.1. Let $F:[0,1]^{3} \rightarrow \mathbb{R}$ be a quadratic function of the form (4) and $I:[0,1]^{2} \rightarrow[0,1]$ a fuzzy implication function that satisfies (NP) and (OP). If $I_{F}$ fulfils (NP) and (OP), then $f+c=0$ and $d=0$.

Moreover, in this case, $F$ is given by

$$
\begin{aligned}
F_{\alpha, \beta}(x, y, z)= & \alpha z^{2}-\beta x z-\alpha y z+\beta x+\alpha y+ \\
& (1-\alpha+\beta) z-\beta
\end{aligned}
$$

where $\alpha, \beta \in \mathbb{R}$.

Proof. If $I_{F}$ satisfies (NP) then according to Proposition 3.2-(i) we obtain the condition $f+c=0$. Moreover, it is well known that (OP) implies (IP). Consequently, since $I$ satisfies (OP), it satisfies also (IP) and we obtain $d=0$, by applying Proposition 3.2-(ii). In this case, taking $\alpha=c$ and $\beta=g$, it is clear that $F$ is given by Expression (5).

Remark 4.1. Note that although $I_{F_{\alpha, \beta}}$ satisfies the additional properties (NP) and (OP), it is not always a fuzzy implication function. For instance, if we consider 
$\alpha=0$ and $\beta=2$ in Expression (5) and the Gödel implication

$$
I_{\mathbf{G D}}(x, y)= \begin{cases}1 & \text { if } x \leq y, \\ y & \text { if } x>y,\end{cases}
$$

we obtain

$$
\left(I_{\mathbf{G D}}\right)_{F_{0,2}}(x, y)= \begin{cases}1 & \text { if } x \leq y, \\ -2 x y+2 x+3 y-2 & \text { if } x>y,\end{cases}
$$

which is not a fuzzy implication function since for all $x>y$, we have that $\frac{\partial\left(I_{\mathbf{G D}}\right)_{F_{0,2}}(x, y)}{\partial x}=2-2 y>0$ and then $\left(I_{\mathbf{G D}}\right)_{F_{0,2}}$ is not decreasing in the first variable.

Now let us investigate when $I_{F_{\alpha, \beta}}$ is a fuzzy implication function in the sense of Definition 2.2.

Theorem 4.1. Let $F:[0,1]^{3} \rightarrow \mathbb{R}$ be a quadratic function of the form (2) and $I_{F}$ the binary function given by (3). Suppose that $I_{F}=I_{\alpha, \beta}$, where

$$
\begin{aligned}
I_{\alpha, \beta}(x, y)= & \alpha I(x, y)^{2}-\beta x I(x, y)-\alpha y I(x, y)+\beta x+ \\
& \alpha y+(1-\alpha+\beta) I(x, y)-\beta
\end{aligned}
$$

with $\alpha$, $\beta$ such that $-1 \leq \beta \leq 0$ and $0 \leq \alpha \leq 1+\beta$. Then for each I in $\mathscr{I}_{\mathbf{N P}, \mathbf{O P}}$, the function $I_{\alpha, \beta}$ is also in $\mathscr{I}_{\mathbf{N P}, \mathbf{O P}}$, that is, $I_{\alpha, \beta}$ is a fuzzy implication function that fulfils (NP) and (OP).

Proof. Let us denote by $P$ the region in $\mathbb{R}^{2}$ of all pairs $(\alpha, \beta)$ delimited by the restrictions given in the theorem and depicted in Figure 1. Due to the convexity of $P$, in order to prove the theorem, we only need to prove that functions $I_{0,0}, I_{1,0}$ and $I_{0,-1}$, corresponding to the vertices of the region $P$, are fuzzy implication functions satisfying (NP) and (OP).

- The function corresponding to the vertex $(0,0)$ is given by $I_{0,0}(x, y)=I(x, y)$, which is by hypothesis a fuzzy implication function satisfying (NP) and (OP).

- The case corresponding to the vertex $(0,-1)$ is given by the function $I_{0,-1}(x, y)=x I(x, y)-x+1$. Let us consider any $x \in[0,1]$ and $\delta>0$, such that $x+\delta \in$ $[0,1]$, and any fixed $y \in[0,1]$. We have that

$$
\begin{aligned}
& I_{0,-1}(x+\delta, y)-I_{0,-1}(x, y) \\
& =(x+\delta) I(x+\delta, y)-(x+\delta)+1-x I(x, y)+x-1 \\
& =x I(x+\delta, y)+\delta I(x+\delta, y)-\delta-x I(x, y) \\
& =x(I(x+\delta, y)-I(x, y))+\delta(I(x+\delta, y)-1) \leq 0 .
\end{aligned}
$$

Last inequality is due to the fact that $I(x+\delta, y)-$ $I(x, y) \leq 0$ and $I(x+\delta, y)-1 \leq 0$ since $I$ is a fuzzy implication function. Now, let us consider any $y \in[0,1]$ and $\delta>0$, such that $y+\delta \in[0,1]$, and any fixed $x \in[0,1]$. We have that

$$
I_{0,-1}(x, y+\delta)-I_{0,-1}(x, y)=x(I(x, y+\delta)-I(x, y)) \geq 0
$$

because $I(x, y+\delta)-I(x, y) \geq 0$ due to the fact that $I$ is increasing in the second variable. Therefore, since (I3) is also satisfied, $I_{0,-1}$ is a fuzzy implication function. Now, by Proposition 3.2-(i), it satisfies (NP). To prove (OP), by Proposition 3.2-(ii), it satisfies (IP) and with the monotonicities, $I_{0,-1}(x, y)=1$ for all $x \leq y$. Finally, it is easy to prove that $0 \leq I_{0,-1}(x, y)=x I(x, y)-x+$ $1<1$ for all $x>y$, thus fulfilling (OP).

- Finally, the case corresponding to the vertex $(1,0)$ is given by the function $I_{1,0}(x, y)=I^{2}(x, y)-y I(x, y)+y$. Let us consider any $x \in[0,1]$ and $\delta>0$, such that $x+$ $\delta \in[0,1]$, and any fixed $y \in[0,1]$. We have that

$$
\begin{aligned}
& I_{1,0}(x+\delta, y)-I_{1,0}(x, y)=I^{2}(x+\delta, y)-y I(x+\delta, y) \\
& +y-I^{2}(x, y)+y I(x, y)-y=(I(x+\delta, y)-I(x, y)) . \\
& (-y+I(x, y)+I(x+\delta, y)) \leq 0 .
\end{aligned}
$$

Last inequality is due to the fact that $I$ is decreasing in the first variable and that it satisfies (NP) and consequently, $I(x, y) \geq y$. Now, let us consider any $y \in[0,1]$ and $\delta>0$, such that $y+\delta \in[0,1]$, and any fixed $x \in[0,1]$. We have that

$$
\begin{aligned}
& I_{1,0}(x, y+\delta)-I_{1,0}(x, y)=I^{2}(x, y+\delta)-(y+\delta) . \\
& I(x, y+\delta)+y+\delta-I^{2}(x, y)+y I(x, y)-y= \\
& (I(x, y+\delta)-I(x, y)) \cdot(I(x, y)-y)+ \\
& \delta(1-I(x, y+\delta))+\left(I^{2}(x, y+\delta)-I(x, y+\delta) .\right. \\
& I(x, y)) \geq 0 .
\end{aligned}
$$

Last inequality is due to the fact that $I$ is increasing in the second variable and that it satisfies (NP).

Analogously to the previous case, $I_{1,0}$ satisfies (NP) and $I_{1,0}(x, y)=1$ for all $x \leq y$. We must prove that $I_{1,0}(x, y)=I^{2}(x, y)-y I(x, y)+y<1$ for all $x>y$. However, this is equivalent to show $I^{2}(x, y)-y I(x, y)<$ $1-y$ for all $x>y$. Now, if we have in mind that function $I(x, y)(I(x, y)-y)$ with the conditions $0 \leq y \leq$ $I(x, y) \leq 1$ takes values between 0 (when $I(x, y)=y$ ) and $1-y($ when $I(x, y)=1)$ the result follows immediately.

Finally, due to the convexity of the set of eligible parameter values $P$, each point $(\alpha, \beta) \in P$ is a convex combination of the vertices of $P$. The fuzzy implication function $I_{\alpha, \beta}$ corresponding to the point $(\alpha, \beta)$ can be expressed as the same convex combination of fuzzy implication functions $I_{0,0}, I_{0,-1}$ and $I_{1,0}$ corresponding to the vertices of $P$. As the family of all fuzzy implication functions is a convex set $[4,5], I_{\alpha, \beta}$ is a fuzzy implication function and the theorem is proved. Note that the convex combination preserves (NP) and (OP)

Theorem 4.1 gives a sufficient condition for our purposes, let us now provide a necessary one in the following result. 
Theorem 4.2. Let $F:[0,1]^{3} \rightarrow \mathbb{R}$ be a quadratic function of the form (2) and $I_{F}$ the binary function given by (3). Suppose that for each I in $\mathscr{I}_{\mathbf{N P}, \mathbf{O P}}$, the function $I_{F}$ is also in $\mathscr{I}_{\mathbf{N P}, \mathbf{O P}}$. Then $I_{F}=I_{\alpha, \beta}$ given by Eq. (6) with $\alpha$, $\beta$ such that $-1 \leq \beta \leq 0,-1 \leq \alpha+\beta \leq 1$ and $\alpha \geq 0$.

Proof. Let us denote by $P \cup P^{\prime}$ the region of all pairs $(\alpha, \beta)$ delimited by the restrictions of parameters given in the theorem (see Figure 2) and suppose that for each $I$ in $\mathscr{I}_{\mathbf{N P}, \mathbf{O P}}$, the function $I_{F}$ is also in $\mathscr{I}_{\mathbf{N P}, \mathbf{O P}}$. First of all, we already know from Proposition 4.1 that the quadratic function $F$ is of the form (5) and consequently $I_{F}$ is an $I_{\alpha, \beta}$ of the form (6). Let us see that parameters $\alpha, \beta$ are in $P \cup P^{\prime}$ through some steps.

- Let us consider $I$ as the Gödel implication function. It is clear that $I_{\mathbf{G D}} \in \mathscr{I}_{\mathbf{N P}, \mathbf{O P}}$. A simple computation leads to

$$
I_{\alpha, \beta}(x, y)=-\beta x y+y(1+\beta)+\beta x-\beta
$$

for all $x>y$. Moreover, it must fulfil that $0 \leq$ $I_{\alpha, \beta}(x, y)<1$ for all $x>y$ and, in particular, $0 \leq$ $I_{\alpha, \beta}(x, 0)<1$ which leads to $-1 \leq \beta \leq 0$.

- Similarly, the Fodor implication, which is given by

$$
I_{\mathbf{F D}}(x, y)= \begin{cases}1 & \text { if } x \leq y, \\ \max \{1-x, y\} & \text { otherwise, }\end{cases}
$$

is in $\mathscr{I}_{\mathbf{N P}, \mathbf{O P}}$ and so is the corresponding $I_{\alpha, \beta}$. Again, a straightforward computation shows that

$$
I_{\alpha, \beta}(x, y)=(\alpha+\beta) x^{2}-(\alpha+\beta) x+\alpha x y-x+1
$$

for all $x>y$ and $1-x \geq y$. First, this operator must be decreasing in the first variable. This implies that $2(\alpha+\beta) x-1-(\alpha+\beta)+\alpha y \leq 0$ for all $x>y$ and $1-x \geq y$ implying that $-1 \leq \alpha+\beta \leq 1$. Second, this operator must be increasing with respect to the second variable, which leads to $\alpha x \geq 0$ for all $x \in[0,1]$ implying that $\alpha \geq 0$.

Joining Theorems 4.1 and 4.2 the characterization of the whole region of parameter values which provide quadratic construction methods of fuzzy implication functions preserving (NP) and (OP) is not obtained. Indeed, we only derive that such region must be between $P$ and $P^{\prime}$ (see again Figure 2). Note that the region would be characterized by $P^{\prime}$ if the function $I_{2,-1}$ corresponding to the vertex $(2,-1)$ is a fuzzy implication function. This is still an open problem. On the contrary, if such $I_{2,-1}$ is not a fuzzy implication function then more restrictions would be necessary to characterize the region between $P$ and $P^{\prime}$.

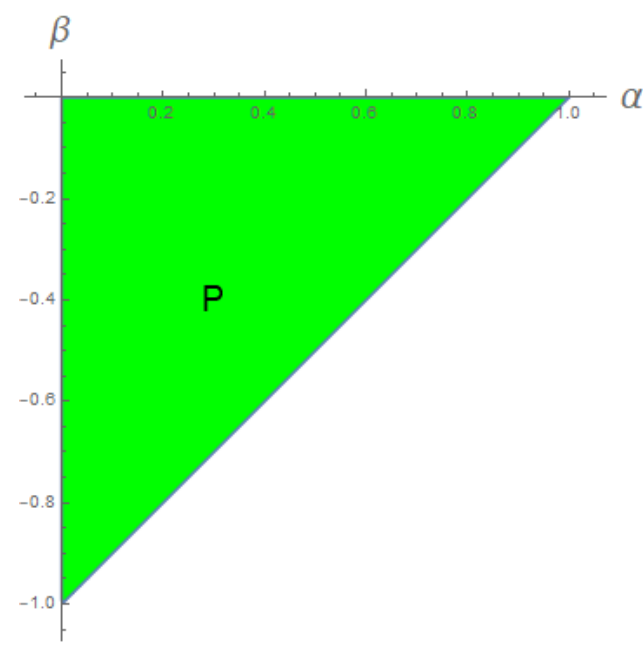

Figure 1: A set $P$ of eligible pairs of coeficients $(\alpha, \beta)$ for the quadratic construction of fuzzy implication functions preserving (NP) and (OP).

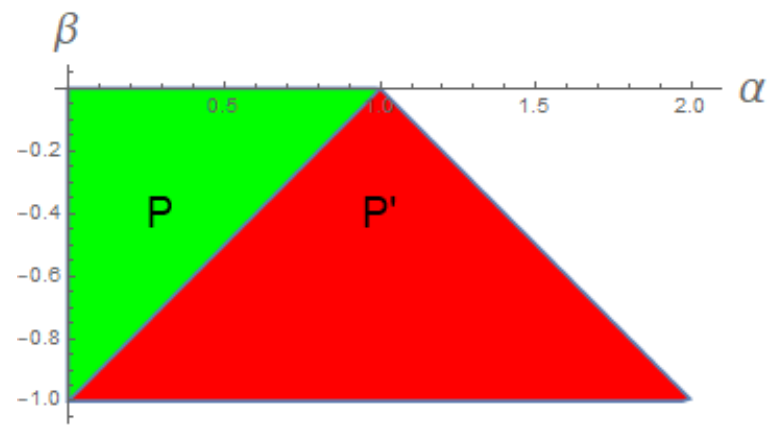

Figure 2: Set $P$ already described in Figure 1 jointly with set $P^{\prime}$ of potential eligible pairs of coefficients $(\alpha, \beta)$ for the quadratic construction of fuzzy implication functions preserving (NP) and (OP).

\subsection{Quadratic constructions preserving $\left(\mathbf{C P}\left(N_{c}\right)\right)$ and $(\mathrm{OP})$}

We will perform a similar analysis to the one carried out in the previous subsection. This first result gives some conditions on the parameters of the quadratic function of the form (4) when function $I_{F}$ satisfies $\left(\mathbf{C P}\left(N_{c}\right)\right)$ and $(\mathbf{O P})$.

Proposition 4.2. Let $F:[0,1]^{3} \rightarrow \mathbb{R}$ be a quadratic function of the form (4) and $I:[0,1]^{2} \rightarrow[0,1]$ be a fuzzy implication function that satisfies $\left(\mathbf{C P}\left(N_{c}\right)\right)$ and (OP). If $I_{F}$ fulfils $\left(\mathbf{C P}\left(N_{C}\right)\right)$ and $(\mathbf{O P})$ then $d+g-f=0$ and $d=0$.

Moreover, in this case, $F$ is given by

$$
\begin{aligned}
F_{\alpha, \beta}(x, y, z)= & \alpha z^{2}-\beta x z+\beta y z+\beta x-\beta y \\
& +(1-\alpha+\beta) z-\beta
\end{aligned}
$$

where $\alpha, \beta \in \mathbb{R}$. 
Proof. Similar to the proof of Proposition 4.1 by using Proposition 3.2-(ii) and (iii).

Remark 4.2. Note that although $I_{F_{\alpha, \beta}}$ satisfies $\left(\mathbf{C P}\left(\mathbf{N}_{\mathbf{c}}\right)\right)$ and $(\mathbf{O P})$ it is not always a fuzzy implication function. For example, if we consider $\alpha=0$ and $\beta=3$ in Expression (7) and we apply the method to the Rescher implication $I_{\mathbf{R S}}$, given by

$$
I_{\mathbf{R S}}(x, y)= \begin{cases}1 & \text { if } x \leq y \\ 0 & \text { if } x>y\end{cases}
$$

which satisfies $\left(\mathbf{C P}\left(\mathbf{N}_{\mathbf{c}}\right)\right)$ and $(\mathbf{O P})$, we obtain

$$
\left(I_{\mathbf{R S}}\right)_{F_{0,3}}(x, y)= \begin{cases}1 & \text { if } x \leq y, \\ 3(x-y-1) & \text { if } x>y\end{cases}
$$

which is not a fuzzy implication function since $\left(I_{\mathbf{R S}}\right)_{F_{0,3}}\left(\frac{3}{4}, 0\right)=-\frac{3}{4}$.

Let us study when $I_{F_{\alpha, \beta}}$ is a fuzzy implication function that satisfies $\left(\mathbf{C P}\left(N_{c}\right)\right)$ and $(\mathbf{O P})$.

Theorem 4.3. Let $F:[0,1]^{3} \rightarrow \mathbb{R}$ be a quadratic function of the form (2) and $I_{F}$ the binary function given by (3). Suppose that $I_{F}=I_{\alpha, \beta}$, where

$$
\begin{aligned}
I_{\alpha, \beta}(x, y)= & \alpha I(x, y)^{2}-\beta x I(x, y)+\beta y I(x, y)+\beta x-\beta y \\
& +(1-\alpha+\beta) I(x, y)-\beta
\end{aligned}
$$

with $\alpha$, $\beta$ such that $-1 \leq \beta \leq 0,1+\beta \leq \alpha \leq-1-$

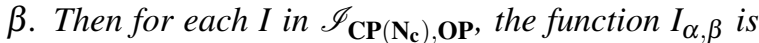

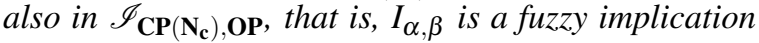
function that fulfils $\left(\mathbf{C P}\left(N_{c}\right)\right)$ and $(\mathbf{O P})$.

Proof. Let $P$ denote the region of all pairs $(\alpha, \beta)$ embedded by the restrictions given in the theorem (see Figure 3 ). Due to the convexity of $P$, in order to prove the result we only need to check that the operators $I_{-1,0}, I_{0,-1}$ and $I_{1,0}$ corresponding to the vertices of the region $P$ are fuzzy implication functions satisfying $\left(\mathbf{C P}\left(N_{c}\right)\right)$ and (OP). We will show it step by step.

- The case corresponding to the vertex $(-1,0)$ is given by the function $I_{-1,0}(x, y)=-I^{2}(x, y)+2 I(x, y)$. Let us check the monotonicities. Let us consider any $x \in$ $[0,1]$ and $\delta>0$, such that $x+\delta \in[0,1]$, and any fixed $y \in[0,1]$. We have that

$$
\begin{aligned}
& I_{-1,0}(x+\delta, y)-I_{-1,0}(x, y) \\
& =-I^{2}(x+\delta, y)+2 I(x+\delta, y)+I^{2}(x, y)-2 I(x, y) \\
& =(I(x+\delta, y)-I(x, y))(2-I(x, y)) \\
& +\left(I(x, y) I(x+\delta, y)-I^{2}(x+\delta, y)\right) \leq 0 .
\end{aligned}
$$

The last inequality is due to the fact that $I$ is decreasing in the first variable. Now, let us consider any $y \in[0,1]$ and $\delta>0$, such that $y+\delta \in[0,1]$, and any fixed $x \in$ $[0,1]$. We have that

$$
\begin{aligned}
& I_{-1,0}(x, y+\delta)-I_{-1,0}(x, y) \\
& =-I^{2}(x, y+\delta)+2 I(x, y+\delta)+I^{2}(x, y)-2 I(x, y) \\
& =(I(x, y+\delta)-I(x, y))(1-I(x, y)) \\
& +(I(x, y+\delta)-I(x, y))((1-I(x, y+\delta)) \geq 0 .
\end{aligned}
$$

The last inequality is due to the fact that $I$ is increasing in the second variable. Therefore, since (I3) is also satisfied, $I_{-1,0}$ is a fuzzy implication function. Now, by Proposition 3.2-(iii), it satisfies $\left(\mathbf{C P}\left(N_{c}\right)\right)$. To prove (OP), by Proposition 3.2-(ii), it satisfies (IP) and with the monotonicities, $I_{-1,0}(x, y)=1$ for all $x \leq y$. Finally, it is easy to prove that $0 \leq I_{-1,0}(x, y)=-I^{2}(x, y)+$ $2 I(x, y)<1$ for all $x>y$, thus fulfilling (OP).

- The case corresponding to the vertex $(0,-1)$ is provided by the function $I_{0,-1}(x, y)=x I(x, y)-y I(x, y)-$ $x+y+1$. Analogously, to the previous case, $I_{0,-1}$ satisfies $\left(\mathbf{C P}\left(N_{c}\right)\right)$ and $I_{0,-1}(x, y)=1$ for all $x \leq y$. Finally, it is clear that $0 \leq I_{0,-1}(x, y)=x I(x, y)-y I(x, y)-x+$ $y+1<1$ for all $x>y$ since it is equivalent to show that $(x-y)(I(x, y)-1)<0$ for all $x>y$. Thus (OP) is satisfied.

Now, we will prove the monotonicities. Let us consider any $x \in[0,1]$ and $\delta>0$, such that $x+\delta \in[0,1]$, and any fixed $y \in[0,1]$ such that $x>y$. We have that

$$
\begin{aligned}
& I_{0,-1}(x+\delta, y)-I_{0,-1}(x, y) \\
& =(x+\delta) I(x+\delta, y)-y I(x+\delta, y)-x-\delta+y+1 \\
& -x I(x, y)+y I(x, y)+x-y-1 \\
& =(x-y)(I(x+\delta, y)-I(x, y))+\delta(I(x+\delta, y)-1) \\
& \leq 0
\end{aligned}
$$

The last inequality is due to the fact that $I$ is decreasing in the first variable and $x>y$. Let us check the other monotonicity. Let us consider any $y \in[0,1]$ and $\delta>0$, such that $y+\delta \in[0,1]$, and any fixed $x \in[0,1]$ such that $x>y$. We have that

$$
\begin{aligned}
& I_{0,-1}(x, y+\delta)-I_{0,-1}(x, y) \\
& =x I(x, y+\delta)-(y+\delta) I(x, y+\delta)-x+y+\delta+1 \\
& -x I(x, y)+y I(x, y)+x-y-1 \\
& =(x-y)(I(x, y+\delta)-I(x, y))+\delta(1-I(x, y+\delta)) \\
& \geq 0
\end{aligned}
$$

The last inequality is due to the fact that $I$ is increasing in the second variable and $x>y$.

- The case corresponding to the vertex $(1,0)$ is given by the function $I_{1,0}(x, y)=I^{2}(x, y)$ that straightforwardly it is a fuzzy implication function that satisfies $\left(\mathbf{C P}\left(\mathbf{N}_{\mathbf{c}}\right)\right)$ and $(\mathbf{O P})$ when $I$ also satisfy them.

Due to the convexity of the set of eligible parameter values $P$, each point $(\alpha, \beta) \in P$ is a convex combination of the vertices of $P$. The fuzzy implication 


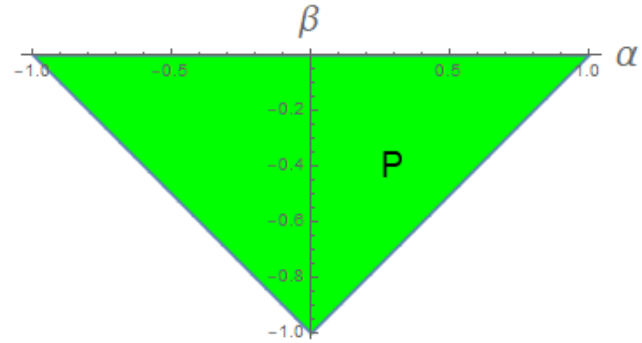

Figure 3: A set $P$ of eligible pairs of coefficients $(\alpha, \beta)$ for the quadratic construction of fuzzy implications preserving $\left(\mathbf{C P}\left(N_{c}\right)\right)$ and $(\mathbf{O P})$.

function $I_{\alpha, \beta}$ corresponding to the point $(\alpha, \beta)$ can be expressed as the same convex combination of fuzzy implication functions $I_{-1,0}, I_{0,-1}$ and $I_{1,0}$ corresponding to the vertices of $P$. As the family of all fuzzy implication functions is a convex set $[4,5], I_{\alpha, \beta}$ is a fuzzy implication function and the theorem is proved. Note that the convex combination preserves (OP) and $\left(\mathbf{C P}\left(N_{c}\right)\right)$.

In a similar way as it was done in the previous subsection, now we want to investigate when $I_{F_{\alpha \beta}}$ is a fuzzy implication function that satisfies $\left(\mathbf{C P}\left(N_{c}\right)\right)$ and (OP). While Theorem 4.3 provides a sufficient condition for our purposes, let us now present a necessary one in the following result.

Theorem 4.4. Let $F:[0,1]^{3} \rightarrow \mathbb{R}$ be a quadratic function of the form (2) and $I_{F}$ the binary function given

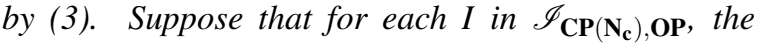

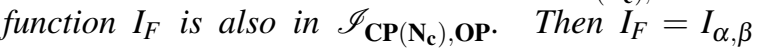
given by Eq. (8) with $\alpha, \beta$ such that $-1 \leq \beta \leq 0$ and $-1 \leq \alpha+\beta \leq 1$.

Proof. Let us denote by $P \cup P^{\prime}$ the region of all pairs $(\alpha, \beta)$ bounded by the restrictions of parameters given in the theorem (see Figure 4) and suppose that for each

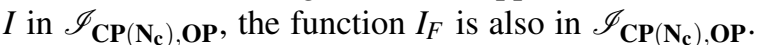
First of all we already know from Proposition 4.2 that the quadratic function $F$ is of the form (7) and consequently $I_{F}$ is an $I_{\alpha, \beta}$ of the form (8). Let us see that parameters $\alpha, \beta$ are in $P \cup P^{\prime}$ through some steps.

- Let us consider $I$ the Rescher implication $I_{\mathbf{R S}}$. It is obvious that $I_{\mathbf{R S}} \in \mathscr{I}_{\mathbf{C P}\left(\mathbf{N}_{\mathbf{c}}\right) \text {,OP }}$ and we get that

$$
I_{\alpha, \beta}(x, y)=\beta(x-y-1)
$$

for all $x>y$. Now, $0 \leq I_{\alpha, \beta}(x, y)<1$ must hold for all $x>y$, that is, $0 \leq \beta(x-y-1)<1$ which implies $-1 \leq \beta \leq 0$.

- Similarly, the Fodor implication is in $\mathscr{I}_{\mathbf{C P}\left(\mathbf{N}_{\mathbf{c}}\right), \mathbf{O P}}$ and so is the corresponding $I_{\alpha, \beta}$. In this case, we

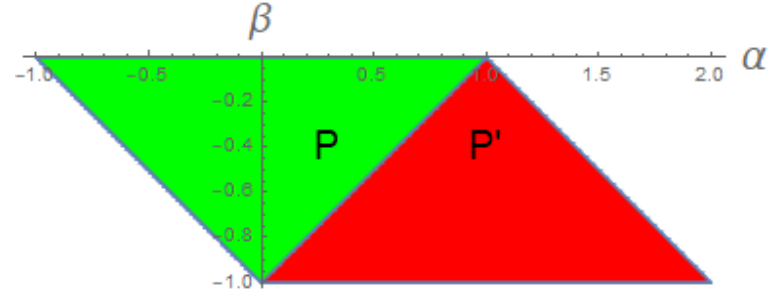

Figure 4: Set $P$ already described in Figure 3 jointly with set $P^{\prime}$ of potential eligible pairs of coefficients $(\alpha, \beta)$ for the quadratic construction of fuzzy implication functions preserving $\left(\mathbf{C P}\left(N_{c}\right)\right)$ and (OP).

have that

$$
I_{\alpha, \beta}(x, y)=(\alpha+\beta) x^{2}-(\alpha+\beta) x-\beta x y-x+1
$$

for all $x>y$ and $1-x \geq y$. This operator must be decreasing with respect to the first variable, that is, $2 x(\alpha+\beta)-(\alpha+\beta)-\beta y-1 \leq 0$ must hold for all $x>y$ and $1-x \geq y$. In particular, if we take $y=0$ we have $2 x(\alpha+\beta)-(\alpha+\beta)-1 \leq 0$ which implies that $-1 \leq \alpha+\beta \leq 1$.

Joining Theorems 4.3 and 4.4, unfortunately again the characterization of the whole region of parameter values which provide quadratic construction methods of fuzzy implication functions preserving $\left(\mathbf{C P}\left(N_{c}\right)\right)$ and (OP) is not obtained. Such a region must be between $P$ and $P^{\prime}$ (see again Figure 4).

\section{Conclusions and Future Work}

In this paper, the preservation of pairs of additional properties via the construction method of fuzzy implication functions from a given one and a quadratic polynomial functions started in [7] has been resumed. In particular, the cases corresponding to the preservation of (i) (NP) and (OP), (ii) $\left(\mathbf{C P}\left(N_{c}\right)\right)$ and (OP) have been investigated. In both cases, sufficient conditions to ensure the preservation are given, but the full characterization of the quadratic construction methods which preserve these pairs of additional properties remain unsolved. Indeed, in both cases, two regions $P \cup P^{\prime}$ of parameter values are presented and the region is sought is between $P$ and $P^{\prime}$.

In the future work, we plan to solve completely these two problems as well as the open problem left in [7] on the preservation of the pair $\left(\mathbf{C P}\left(N_{c}\right)\right)$ and $(\mathbf{N P})$.

\section{Acknowledgement}


This work has been partially supported by the Spanish Grant FEDER/Ministerio de Economía, Industria y Competitividad - AEI/TIN2016-75404-P.

\section{References}

[1] I. Aguiló, J. Suñer, J. Torrens, New types of contrapositivisation of fuzzy implications with respect to fuzzy negations, Inf. Sci. 322 (2015) 223-236.

[2] I. Aguiló, J. Suñer, J. Torrens, A new look on fuzzy implication functions: FNI-implications, in: J. Paulo Carvalho et al. (Ed.), Information Processing and Management of Uncertainty in Knowledge-Based Systems - 16th International Conference, IPMU 2016, Proceedings, Part I, Vol. 610 of Communications in Computer and Information Science, Springer, 2016, pp. 375-386.

[3] M. Baczyński, G. Beliakov, H. Bustince, A. Pradera, Advances in Fuzzy Implication Functions, Vol. 300 of Studies in Fuzziness and Soft Computing Series, Springer-Verlag Berlin Heidelberg, 2013.

[4] M. Baczyński, B. Jayaram, Fuzzy Implications, Vol. 231 of Studies in Fuzziness and Soft Computing, Springer, Berlin Heidelberg, 2008.

[5] M. Baczyński, B. Jayaram, S. Massanet, J. Torrens, Fuzzy implications: Past, present, and future, in: J. Kacprzyk, W. Pedrycz (Eds.), Springer Handbook of Computational Intelligence, Springer Berlin Heidelberg, 2015, pp. 183-202.

[6] J. C. Fodor, M. Roubens, Fuzzy Preference Modelling and Multicriteria Decision Support, Kluwer Academic Publishers, Dordrecht, 1994.

[7] A. Kolesárová, S. Massanet, R. Mesiar, J. V. Riera, J. Torrens, Polynomial constructions of fuzzy implications functions: The quadratic case, Information Sciences 494 (2019) 60-79.

[8] S. Massanet, J. Torrens, On some properties of threshold generated implications, Fuzzy Sets and Systems 205 (2012) 30-49.

[9] S. Massanet, J. Torrens, Threshold generation method of construction of a new implication from two given ones, Fuzzy Sets and Systems 205 (2012) 50-75.

[10] S. Massanet, J. Torrens, On the vertical threshold generation method of fuzzy implication and its properties, Fuzzy Sets and Systems 226 (2013) 32-52.
[11] S. Massanet, J. Torrens, An overview of construction methods of fuzzy implications, in: M. Baczyński, G. Beliakov, H. Bustince, A. Pradera (Eds.), Advances in Fuzzy Implication Functions, Vol. 300 of Studies in Fuzziness and Soft Computing, Springer Berlin Heidelberg, 2013, pp. 1-30.

[12] Y. Shi, B. V. Gasse, D. Ruan, E. Kerre, On dependencies and independencies of fuzzy implication axioms, Fuzzy Sets and Systems 161 (10) (2010) $1388-1405$.

[13] E. Trillas, M. Mas, M. Monserrat, J. Torrens, On the representation of fuzzy rules, Int. J. Approx. Reasoning 48 (2) (2008) 583-597.

[14] N. R. Vemuri, B. Jayaram, Representations through a monoid on the set of fuzzy implications, Fuzzy Sets and Systems 247 (2014) 51 67, theme: Logic and Algebra.

[15] N. R. Vemuri, B. Jayaram, The $\circledast$-composition of fuzzy implications: Closures with respect to properties, powers and families, Fuzzy Sets and Systems 275 (2015) 58-87. 